\title{
A comparative study of the accumulated sialic acid- containing oligosaccharides from cultured human galactosialidosis and sialidosis fibroblasts
}

Johannes van Pelt a, Johannis P. Kamerling a , Johannes F.G. Vliegenthart a , André T. Hoogeveen ${ }^{b}$ and Hans Galjaard ${ }^{b}$

${ }^{a}$ Department of Bio-Organic Chemistry, Utrecht University, Utrecht and ${ }^{b}$ Department of Cell Biology and Genetics, Erasmus University, Rotterdam (The Netherlands)

(Received 18 November 1987; revision received 12 February 1988; accepted after revision 14 February 1988)

Key words: Sialidosis; Galactosialidosis; Sialyloligosaccharides; Lysosomal storage diseases

\section{Summary}

Sialic acid-containing storage material was isolated from cultured human galactosialidosis fibroblasts, by a combination of gel filtration and anion-exchange chromatography on Mono Q. The obtained sialyloligosaccharides were analyzed by $500-\mathrm{MHz}{ }^{1} \mathrm{H}-\mathrm{NMR}$ spectroscopy in combination with sugar analysis and analytical HPLC. The storage material consisted of a series of completely sialylated $N$-acetyllactosamine type of structures having Man $\beta 1-4 \mathrm{GlcNAc}$ at the reducing terminus in common, similar to those recently reported for human sialidosis fibroblasts. Comparison of the storage material from both sources revealed only differences in their relative amounts. In control fibroblasts these compounds could not be detected. The nature of the accumulated compounds is in accordance with the $\alpha$-neuraminidase

\footnotetext{
Abbreviations: HPLC, high-performance liquid chromatography; NMR, nuclear magnetic resonance; Man, mannose; Gal, galactose; Glc, glucose; GlcNAc, $N$-acetylglucosamine; GalNAc, $N$-acetylgalactosamine; NeuAc, $N$-acetylneuraminic acid.

Correspondence to: Prof. Dr. J.F.G. Vliegenthart, Department of Bio-Organic Chemistry, Utrecht University, P.O. Box 80075, NL 3508 TB Utrecht, The Netherlands.
} 
deficiency in both genetic diseases. The additional deficiency of $\beta$-galactosidase in case of galactosialidosis is not reflected in the storage material.

\section{Introduction}

Sialidosis is an autosomal, recessive lysosomal storage disease, caused by an $\alpha$-neuraminidase deficiency. Clinically the deficiency leads to severe symptoms such as mental retardation, skeletal deformities and neurological abnormalities [1]. Galactosialidosis also belongs to the group of lysosomal storage diseases, but is characterized by a deficiency of both $\alpha$-neuraminidase (EC 3.2.1.18) and $\beta$-galactosidase (EC 3.2.1.23) activities [2]. This combined enzyme deficiency is caused by the absence of a $32-\mathrm{kDa}$ protein, which contributes activity to neuraminidase and protects $\beta$-galactosidase against proteolytic degradation [3-6]. The clinical phenotype of galactosialidosis may vary in fashion which is similar to the mild to severe forms of sialidosis [7]. Biochemically, both diseases are further characterized by an excessive excretion of sialyloligosaccharides in urine $[1,7]$. In addition, a considerable increase in the amount of bound sialic acid is found in fibroblasts [8-11]. Recently, we have reported on the isolation and characterization of sialic acid-containing oligosaccharides from sialidosis fibroblasts, showing the presence of completely sialylated mono-, di- and triantennary $\mathrm{N}$-acetyllactosamine type of structures [12]. In this report the sialic acid-containing oligosaccharides accumulated in fibroblasts from a galactosialidosis and a sialidosis patient are compared, in order to obtain information about the influence of the additional $\beta$-galactosidase deficiency on the accumulated carbohydrates in galactosialidosis and about the effect of neuraminidase deficiencies in sialidosis and galactosialidosis.

\section{Materials and methods}

\section{Fibroblasts}

Galactosialidosis fibroblasts were obtained by amniocentesis in a pregnancy at risk. The fetus appeared to be affected. In these cells $\alpha$-neuraminidase activity was almost absent, whereas $5-10 \%$ residual $\beta$-galactosidase activity was measured. All other enzymes tested showed normal activities [13].

Galactosialidosis and control fibroblasts (both 14 Falcon flasks of $75 \mathrm{~cm}^{2}$ ) were grown in HAM's F10 medium (Flow Laboratories, McLean, VA, USA), supplemented with $10 \%$ fetal calf serum. The cells were harvested by trypsinization after 1 wk of confluency, washed several times with phosphate-buffered $(10 \mathrm{mmol} / \mathrm{l})$ saline $(150 \mathrm{mmol} / \mathrm{l})$ and disrupted in distilled water by sonication (two times $10 \mathrm{~s}$ ) at $0^{\circ} \mathrm{C}$. After $10 \mathrm{~min}$ of centrifugation at $10000 \times \mathrm{g}$ at $4^{\circ} \mathrm{C}$, the supernatants were collected and the pellets were washed once more with distilled water. Portions of the supernatants of the galactosialidosis and control fibroblasts were used for de- 
termination of protein and sialic acid concentrations and for sugar analysis. The protein content was determined according to Lowry [14] using bovine serum albumin as a standard. The amounts of free and total sialic acid were measured by the Warren assay, before and after hydrolysis in $0.05 \mathrm{~mol} / 1 \mathrm{H}_{2} \mathrm{SO}_{4}\left(1 \mathrm{~h}, 80^{\circ} \mathrm{C}\right)$, respectively [15]. The remaining part of each supernatant was used for gel permeation chromatography.

\section{Gel permeation chromatography}

The supernatant of galactosialidosis fibroblasts was fractionated on an AcA 202 column $(120 \times 1.6 \mathrm{~cm}, \mathrm{LKB}$, Bromma, Sweden $)$. The elution was performed with $0.17 \mathrm{~mol} / 1 \mathrm{NaCl}$ in $0.05 \mathrm{~mol} / 1$ Tris $/ \mathrm{HCl}$ buffer, $\mathrm{pH} 7.4$, at a flow rate of $18 \mathrm{ml} / \mathrm{h}$. The supernatant of the normal cell line was fractionated on the same column, but

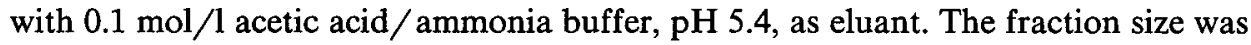
$4.0 \mathrm{ml}$ in both elutions. The fractionations were carried out at room temperature and were monitored by measurement of the UV-absorption at $280 \mathrm{~nm}$ and by hexose determinations with the phenol/sulfuric acid method of Dubois [16].

\section{Anion-exchange chromatography}

Medium-pressure anion-exchange chromatography was carried out on a Mono $Q$ HR 5/5 column utilizing a Fast Protein Liquid Chromatography apparatus (Pharmacia, Uppsala, Sweden). For the separation of sialyloligosaccharides a linear gradient of $0-100 \mathrm{mmol} / 1 \mathrm{NaCl}$ in $10 \mathrm{ml}$ water (Lichrosolv, Merck) at a flow rate of $2.0 \mathrm{ml} / \mathrm{min}$ was applied [17].

The eluate was monitored at $214 \mathrm{~nm}$ using a Pharmacia UV-1/214 detector. For analytical purposes $50-\mu 1$ samples were injected at a 0.1 AU sensitivity setting. For preparative purposes $500 \mu 1$ portions were injected at a sensitivity setting of $1.0 \mathrm{AU}$. Specific fractions were desalted on a Bio-Gel P-2 (100-200 mesh, Bio-Rad) column $(18 \times 1 \mathrm{~cm})$ with distilled water as eluant and subsequently lyophilized.

\section{Sugar analysis}

Sugar analysis was carried out by gas-liquid chromatography on a capillary CP-Sil5 WCOT fused silica column (25 $\mathrm{m} \times 0.32 \mathrm{~mm}$, Chrompack) using a Varian Aerograph 3700 gas chromatograph. The trimethylsilylated methyl glycosides were prepared by methanolysis $\left(1.0 \mathrm{~mol} / 1\right.$ methanolic $\left.\mathrm{HCl}, 24 \mathrm{~h}, 85^{\circ} \mathrm{C}\right), N$-reacetylation and trimethylsilylation [18].

\section{0-MHz ${ }^{1} H-N M R$ spectroscopy}

Sialyloligosaccharides were repeatedly exchanged in ${ }^{2} \mathrm{H}_{2} \mathrm{O}\left(99.96\right.$ atom\% ${ }^{2} \mathrm{H}$, Aldrich) with intermediate lyophilization. ${ }^{1} \mathrm{H}-\mathrm{NMR}$ spectra were recorded on a Bruker WM-500 spectrometer (SON hf-NMR facility, Department of Biophysical Chemistry, University of Nijmegen, The Netherlands) operating at $500 \mathrm{MHz}$ in the Fourier transform mode at a probe temperature of $27^{\circ} \mathrm{C}$ [19]. 


\section{HPLC}

Analytical HPLC was carried out on a Kratos liquid chromatograph equipped with a $10 \mu \mathrm{m}$ Lichrosorb- $\mathrm{NH}_{2}$ column $(250 \times 4.6 \mathrm{~mm}$, Chrompack $)$. The elution of the monosialylated compounds was performed isocratically with a mixture of acetonitrile $/ 30 \mathrm{mmol} / 1 \mathrm{KH}_{2} \mathrm{PO}_{4}, \mathrm{pH} 4.7(66: 34, \mathrm{v} / \mathrm{v})$. The di- and trisialylated compounds were eluted isocratically with a mixture of acetonitrile $/ 30 \mathrm{mmol} / 1$ $\mathrm{KH}_{2} \mathrm{PO}_{4}-\mathrm{K}_{2} \mathrm{HPO}_{4}$ buffer, $\mathrm{pH} 7.0(62.5: 37.5$, v/v). The eluate (flow rate 2.0 $\mathrm{ml} / \mathrm{min}$ ) was monitored by a Spectroflow 783 Programmable Absorbance Detector (Kratos) at $205 \mathrm{~nm}$ and a sensitivity of $0.01 \mathrm{AU}$. Peak areas were calculated by a Spectra Physics SP 4290 Integrator. The HPLC column was calibrated using well-defined sialyloligosaccharides isolated from urine of a sialidosis patient (to be published).

\section{Results}

The quantitative sialic acid determinations in the supernatant of the control and galactosialidosis cell lines are shown in Table I. As is evident from these data, the amount of bound sialic acid per $\mathrm{mg}$ protein is greatly enhanced in case of galactosialidosis as compared to the control value. The quantitative sugar analysis

\section{TABLE I}

Sialic acid determinations (nmol/mg protein) in galactosialidosis (GS), sialidosis (S) and control fibroblasts

\begin{tabular}{lccl}
\hline NeuAc & GS & S & Control \\
\hline Free & 5 & 7 & 6 \\
Bound & 98 & 65 & 6 \\
\hline
\end{tabular}

\section{TABLE II}

Sugar analysis data for galactosialidosis (GS), sialidosis (S) and control fibroblasts (nmol/mg protein)

\begin{tabular}{lrrc}
\hline Monosaccharide & GS & S & Control \\
\hline Man & 233 & 302 & 48 \\
Gal & 340 & 267 & 44 \\
Glc & 1506 & 2666 & 147 \\
GlcNAc & 590 & 406 & 133 \\
GalNAc & 329 & 119 & 211 \\
NeuAc & 186 & 171 & 31 \\
\hline
\end{tabular}

a The large variations in Glc amounts can be caused by different glycogen contents and/or by residual Glc from the culture medium. 


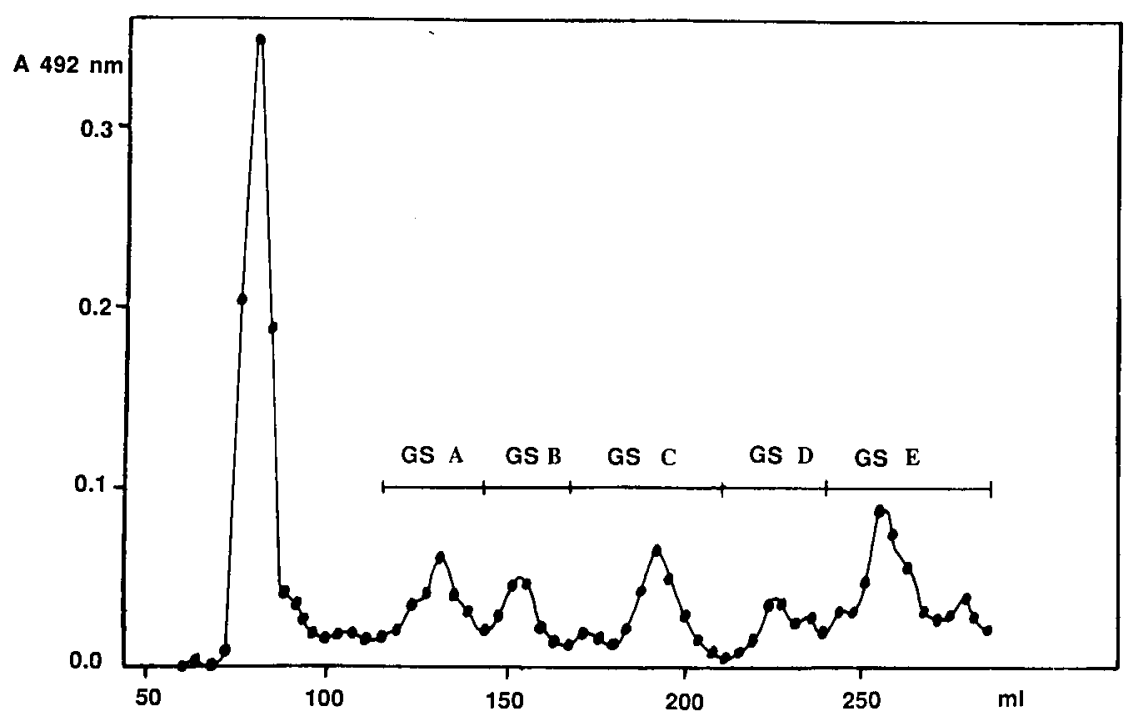

Fig. 1. AcA 202 elution pattern of the supernatant of galactosialidosis fibroblasts, using $0.17 \mathrm{~mol} / 1 \mathrm{NaCl}$

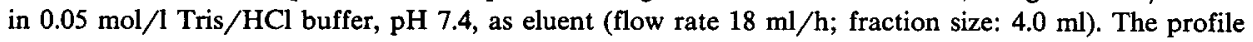
was obtained by phenol/sulfuric acid determinations at $492 \mathrm{~nm}$, using $0.2 \mathrm{ml}$ of the fractions. The fractions GS A-E were pooled as indicated. The fractions GS D and GS $\mathbf{E}$ contained no $N$-glycosidic carbohydrate material.

data, given in Table II, indicate a considerable increase in the amounts of Man, Gal, GlcNAc and NeuAc in the deficient cell line, whereas GalNAc is less increased. For comparison, the data obtained earlier for sialidosis fibroblasts are included in both Tables I and II. The supernatants of the galactosialidosis and control fibroblasts were fractionated by gel filtration on AcA 202. The monitoring of the UV-absorption at $280 \mathrm{~nm}$ showed in both cases one peak in the void volume region of the column. For the galactosialidosis supernatant the elution profile obtained by hexose determinations, showed several small peaks eluting after the void volume (Fig. 1). These peaks were not present in the elution profile of the normal cell line supernatant. For comparison, also the control fractions were pooled in the same way as indicated in Fig. 1. The pooled fractions (GS A-E and N A-E) were desalted and subsequently subjected to anion-exchange chromatography on Mono $\mathrm{Q}$. The profiles of the relevant fractions (GS A-C), monitored by UV-absorption at $214 \mathrm{~nm}$, are given in Fig. 2. The Mono Q column material gives a separation of sialyloligosaccharides mainly according to the number of sialic acid residues [17]. The elution volume of the retarded peak in fraction GS A suggests a trisialylated compound (GS 3), whereas the fractions GS B and GS C seem to contain disialylated (GS 2) and monosialylated (GS 1) compounds, respectively. The control cell line fractions did not contain detectable amounts of sialylated material in the Mono Q chromatographic procedure. 


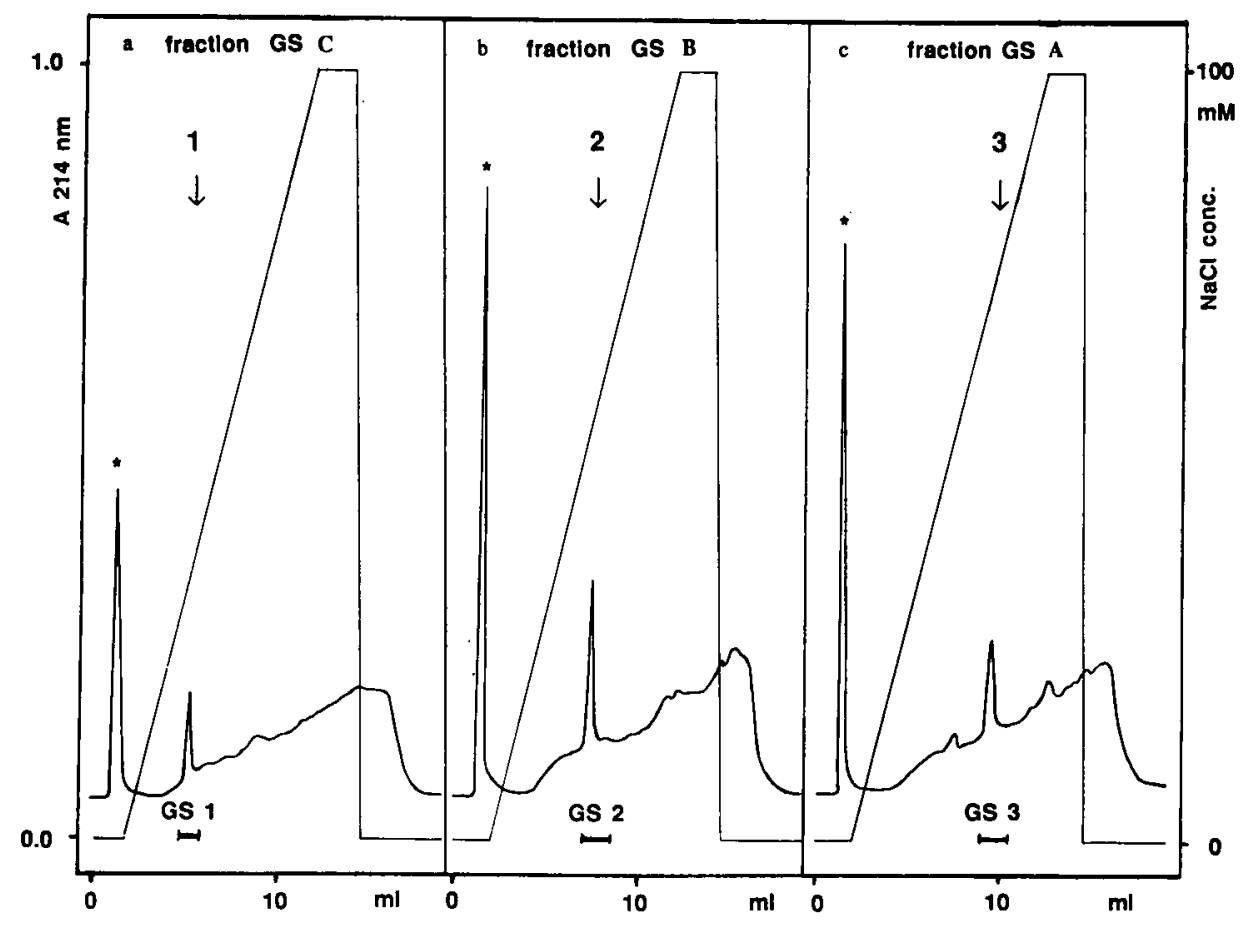

Fig. 2. Mono Q elution profiles of the fractions GS A-C, monitored at $214 \mathrm{~nm}$, using a linear gradient of $0-100 \mathrm{mmol} / 1 \mathrm{NaCl}$ in $10 \mathrm{ml}$ water. The subfractions were pooled as indicated. The peaks designed by an asterisk do not contain carbohydrate material. The numerals above the arrows correspond with the number of sialic acid residues bound in reference compounds isolated from sialidosis urine [17].

Aliquots of the Mono Q subfractions of the galactosialidosis supernatant were subjected to sugar analysis. The results of the fractions GS 1-GS 3 are given in Table III. The neutral Mono $Q$ peaks (designated by ${ }^{a}$ ) contained no detectable amounts of carbohydrate. The data from Table III are indicative for $\mathrm{N}$-glycosidic,

\section{TABLE III}

Sugar analysis data for Mono Q subfractions of the supernatant of galactosialidosis fibroblasts, expressed as molar ratios relative to 3 or 2 Man residues

\begin{tabular}{llll}
\hline Monosaccharide & GS 3 & GS 2 & GS 1 \\
\hline Man & 3 & 3 & 2 \\
Gal & 2.8 & 1.9 & 1.1 \\
GlcNAc & 4.0 & 3.0 & 1.4 \\
NeuAc & 2.5 & 1.7 & 0.8 \\
\hline
\end{tabular}


$N$-acetyllactosamine type of structures with a different degree of branching and number of sialic acid residues.

The fractions GS 1-GS 3 were analyzed by $500-\mathrm{MHz}^{1} \mathrm{H}-\mathrm{NMR}$ spectroscopy in combination with analytical HPLC measurements, according to the same procedure as used for sialidosis fibroblasts [12]. It should be noted that, due to the limited amount of material, it was impossible to perform ${ }^{1} \mathrm{H}-\mathrm{NMR}$ spectroscopy after HPLC separation.

The ${ }^{1} \mathrm{H}$-NMR spectrum of fraction GS 1 showed the presence of a mixture of two monoantennary monosialylated compounds; one is $\alpha 2-6$ sialylated, the other bears NeuAc in an $\alpha 2-3$ linkage:

NeuAc $\alpha 2-3$ Gal $\beta 1-4 G l c N A c \beta 1-2 M a n \alpha 1-3 M a n \beta 1-4 G l c N A c$

NeuAc $\alpha 2-6 \mathrm{Gal} \beta 1-4 \mathrm{GlcNAc} \beta 1-2 \mathrm{Man} \alpha 1-3 \mathrm{Man} \beta 1-4 \mathrm{GlcNAc}$.

The molar ratio of the two isomers is $1: 2(\alpha 2-6: \alpha 2-3)$, which was confirmed by analytical HPLC.

The NMR spectrum of fraction GS 2 revealed the presence of a mixture of disialylated, diantennary compounds. From the spectrum it was clear that both diantennary branches were heterogeneous with respect to the sialic acid linkage types. The analytical HPLC measurements provided conclusive evidence for the precise composition of the mixture. The structures with their molar ratios in parentheses, can be presented as follows:

NeuAc $\alpha 2-3 \mathrm{Gal} \beta 1-4 \mathrm{GlcNAc} \beta 1-2 \mathrm{Man} \alpha 1-6$ NeuAc $\alpha 2-3 \mathrm{Gal} \beta 1-4 \mathrm{GlcNAc} \beta 1-2 \mathrm{Man} \alpha 1-3$ Man $\beta 1-4 \mathrm{GlcNAc}$

NeuAc $\alpha 2-3 \mathrm{Gal} \beta 1-4 \mathrm{GlcNAc} \beta 1-2 \mathrm{Man} \alpha 1-6$

NeuAc $\alpha 2-6 \mathrm{Gal} \beta 1-4 \mathrm{GlcNAc} \beta 1-2 \mathrm{Man} \alpha 1-3>\mathrm{Man} \beta 1-4 \mathrm{GlcNAc}$

NeuAc $\alpha 2-6 \mathrm{Gal} \beta 1-4 \mathrm{GlcNAc} \beta 1-2 \mathrm{Man} \alpha 1-6$

NeuAc $\alpha 2-6 \mathrm{Gal} \beta 1-4 \mathrm{GlcNAc} \beta 1-2 \mathrm{Man} \alpha 1-3-\mathrm{Man} \beta 1-4 \mathrm{GlcNAc}$

Fraction GS 3 contained trisialylated, triantennary structures. From ${ }^{1} \mathrm{H}-\mathrm{NMR}$ spectroscopy and HPLC it was evident that both Gal $\beta 1-4 \mathrm{GlcNAc} \beta 1-2 \mathrm{Man} \alpha 1-3$ and Gal $\beta 1-4$ GlcNAc $\beta 1-2 M a n \alpha 1-6$ branches showed heterogeneity with respect to the NeuAc linkage type, just like in fraction GS 2 . The Gal $\beta 1-4$ GlcNAc $\beta 1-4 M a n \alpha 1-3$ branch was exclusively $\alpha 2-3$ sialylated. The structures with their molar ratios in parentheses can be presented as follows:

NeuAc $\alpha 2-3 \mathrm{Gal} \beta 1-4 \mathrm{GlcNAc} \beta 1-2 \mathrm{Man} \alpha 1-6$,

NeuAc $\alpha 2-3 \mathrm{Gal} \beta 1-4 \mathrm{GlcNAc} \beta 1-2 \mathrm{Man} \alpha 1-3, \mathrm{Man} \beta 1-4 \mathrm{GlcNAc}$

NeuAc $\alpha 2-3 \mathrm{Gal} \beta 1-4 \mathrm{GlcNAc} \beta 1-4$ 
Structures* galactosialidosis sialidosis

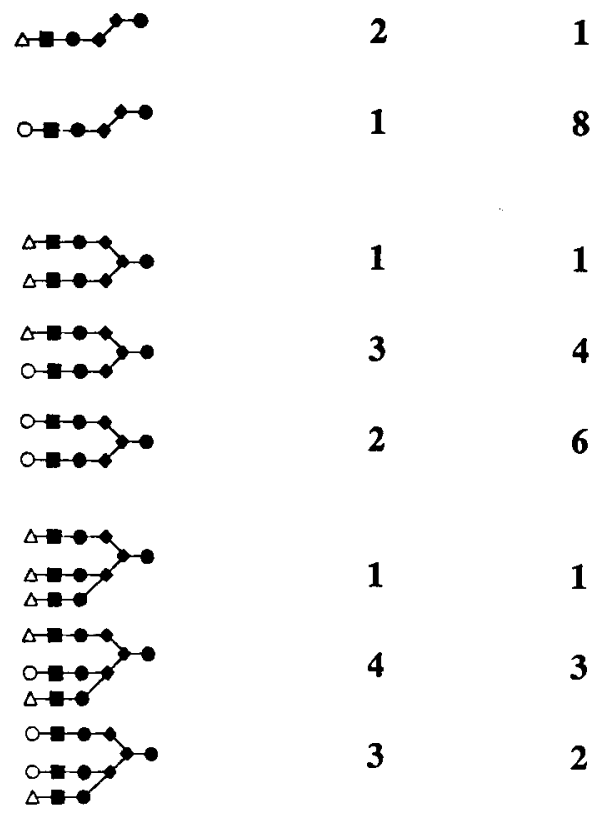

Scheme I. The isolated and analyzed sialyloligosaccharides from galactosialidosis fibroblasts and their molar ratios together with the data from sialidosis fibroblasts [12]. * Symbolic notation: $\Delta$, NeuAc $\alpha 2-3$; O, NeuAc $\alpha 2-6$; $\mathbf{a}, \mathrm{Gal} ; \bullet$, GlcNAc; $\bullet$, Man.

NeuAc $\alpha 2-3 \mathrm{Gal} \beta 1-4 \mathrm{GlcNAc} \beta 1-2 \mathrm{Man} \alpha 1-6$

NeuAc $\alpha 2-6 \mathrm{Gal} \beta 1-4 \mathrm{GlcNAc} \beta 1-2 \mathrm{Man} \alpha 1-3-\mathrm{Man} \beta 1-4 \mathrm{GlcNAc}$

NeuAc $\alpha 2-3$ Gal $\beta 1-4$ GlcNAc $\beta 1-4$

NeuAc $\alpha 2-6$ Gal $\beta 1-4$ GlcNAc $\beta 1-2$ Man $\alpha 1-6$

NeuAc $\alpha 2-6$ Gal $\beta 1-4$ GlcNAc $\beta 1-2 M a n \alpha 1-3-M a n \beta 1-4 G 1 c N A c$

NeuAc $\alpha 2-3 \mathrm{Gal} \beta 1-4 \mathrm{GlcNAc} \beta 1-4$

The analyzed structures from galactosialidosis fibroblasts are summarized in Scheme I, together with the estimated molar ratios. The data obtained for the sialyloligosaccharides isolated from sialidosis fibroblasts [12] are also compiled in Scheme I for comparison.

\section{Discussion}

The observed increases in the amount of bound sialic acid and other sugars in galactosialidosis fibroblasts are caused by the storage of sialyloligosaccharides. The 
structures of the isolated sialic acid-containing carbohydrate chains resemble those from sialidosis urine [20-26] and sialidosis fibroblasts [12]. In all cases heterogeneity in the sialic acid linkage type is observed, indicating the impossibility of removing either $\alpha 2-3-$ or $\alpha 2-6$-linked sialic acid, due to the defective neuraminidase. In this respect a comparison between galactosialidosis and sialidosis storage material reveals only quantitative differences. The most remarkable difference is found for the monoantennary structures, which represent partly digested carbohydrate chains. Another difference is clear from the comparison of diantennary structures from both sources. The isolated sialyloligosaccharides from sialidosis fibroblasts contain relatively more $\alpha 2-6$-bound NeuAc in comparison to those from galactosialidosis. The reason for this is unclear, but it can be caused by a different supply of substrates.

Sialidosis and galactosialidosis have different etiologies. In sialidosis there is a single, primary deficiency of neuraminidase activity, whereas in galactosialidosis deficiency of a $32-\mathrm{kDa}$ protein causes deficiencies of both neuraminidase and $\beta$-galactosidase [3-6]. According to our results, these different primary defects lead to the same kind of storage material in fibroblasts. All the branches of the isolated structures are sialylated and no terminal galactose is detected. This means that the $\beta$-galactosidase deficiency in galactosialidosis is not reflected in the storage material. One possible explanation for this phenomenon is that the residual $\beta$-galactosidase activity is high enough to catabolize carbohydrate chains with terminal galactose. Another possibility is that the amount of carbohydrate chains ending on galactose is fairly low, compared to chains terminating with sialic acid. The galactosidase deficiency would then remain obscure as the catabolism of the majority of carbohydrate chains stops at the level of sialic acid.

The approach outlined in this report can be of practical use in screening procedures. It is possible to detect quantitative differences in carbohydrate amounts by sugar analysis, using aliquots of the supernatant from fibroblasts. Moreover, it is possible to perform structural investigations on accumulated carbohydrate containing material in fibroblasts. The combination of ${ }^{1} \mathrm{H}-\mathrm{NMR}$ spectroscopy and analytical HPLC with suitable reference compounds offers a sensitive and accurate method for this purpose.

\section{Acknowledgements}

The authors wish to thank Dr. J.A. van Kuik for recording the ${ }^{1} \mathrm{H}-\mathrm{NMR}$ spectra and Dr. A.J.J. Reuser for helpfull discussions. This investigation was supported by The Netherlands. Foundation for Chemical Research (SON) with financial aid from The Netherlands Organization for the Advancement of Pure Research (ZWO) and by the Foundation of Clinical Genetics, Rotterdam (The Netherlands).

\section{References}

1 Lowden JA, O'Brien JS. Sialidosis: a review of human neuraminidase deficiency. Am J Hum Genet 1979;31:1-18. 
2 Wenger DA, Tarby TJ, Wharton C. Macular cherry red spots and myoclonus with dementia: coexistent neuraminidase and $\beta$-galactosidase deficiencies. Biochem Biophys Res Commun 1978;82:589-595.

3 Hoogeveen AT, D'Azzo A, Brossmer R, Galjaard H. Correction of combined $\beta$-galactosidase/ neuraminidase deficiency in human fibroblasts. Biochem Biophys Res Commun 1981;103:292-299.

4 Van Diggelen OP, Hoogeveen AT, Smith PJ, Reuser AJJ, Galjaard H. Enhanced proteolytic degradation of normal $\beta$-galactosidase in the lysosomal storage disease with combined $\beta$-galactosidase and neuraminidase deficiency. Biochim Biophys Acta 1982;703:69-76.

5 D'Azzo A, Hoogeveen AT, Reuser AJJ, Robinson D, Galjaard H. Molecular defect in combined $\beta$-galactosidase and neuraminidase deficiency in man. Proc Natl Acad Sci USA 1982;79:4535-4539.

6 Verheijen FW, Palmeri S, Hoogeveen AT, Galjaard H. Human placental neuraminidase. Activation, stabilization and association with $\beta$-galactosidase and its 'protective' enzyme. Eur $\mathrm{J}$ Biochem 1985; 149:315-321.

7 Suzuki Y, Sakuraba H, Yamanaka T, Ko Y-M, Iimori Y, Okamura Y, Hoogeveen AT. Galactosialidosis: a comparative study of clinical and biochemical data on 22 patients. In: Arima M, Suzuki Y, Yabuuchi H, eds. The developing brain and its disorders. Tokyo: Univ. Tokyo Press, 1984;161-175.

8 Thomas GH, Tiller Jr GE, Reynolds LW, Miller CS, Blace JW. Increased levels of sialic acid associated with a sialidase deficiency in I-cell disease (mucolipidosis II) fibroblasts. Biochem Biophys Res Commun 1976;71:188-195.

- 9 Cantz M, Gehler J, Spranger J. Mucolipidosis I: increased sialic acid content and deficiency of an $\alpha-N$-acetylneuraminidase in cultured fibroblasts. Biochem Biophys Res Commun 1977;74:732-738.

10 Kelly TE, Graetz G. Isolated acid neuraminidase deficiency: a distinct lysosomal storage disease. Am J Med Genet 1977;1:31-46.

11 Aylsworth AS, Thomas GH, Hood JL, Malouf N, Libert J. A severe infantile sialidosis: clinical, biochemical and microscopic features. J Pediat 1980;96:662-668.

12 Van Pelt J, Kamerling JP, Vliegenthart JFG, Verheijen FW, Galjaard H. Isolation and structural characterization of sialic acid-containing storage material from mucolipidosis I (sialidosis) fibroblasts. Biochim Biophys Acta 1988;in press.

13 Kleijer WJ, Hoogeveen A, Verheijen FW, et al. Prenatal diagnosis of sialidosis with combined neuraminidase and $\beta$-galactosidase deficiency. Clin Genet 1979;16:60-61.

14 Lowry $\mathrm{OH}$, Rosebrough NJ, Farr AL, Randall RJ. Protein measurement with the folin phenol reagent. J Biol Chem 1951;193:265-275.

15 Warren L. The thiobarbituric acid assay of sialic acids. J Biol Chem 1959;234:1971-1975.

16 Dubois M, Gilles KA, Hamilton JK, Rebers PA, Smith F. Colorimetric method for determining of sugars and related substances. Anal Chem 1956;28:350-356.

17 Van Pelt J, Damm JBL, Kamerling JP, Vliegenthart JFG. Separation of sialyl-oligosaccharides by medium-pressure anion-exchange chromatography on Mono Q. Carbohydr Res 1987;169:43-51.

18 Kamerling JP, Vliegenthart JFG. Gas-liquid chromatography and mass spectrometry of sialic acids. Cell Biol Monogr 1982;10:95-125.

19 Vliegenthart JFG, Dorland L, Van Halbeek H. High-resolution ${ }^{1} \mathrm{H}$-nuclear magnetic resonance spectroscopy as a tool in the structural analyis of carbohydrates related to glycoproteins. Adv Carbohydr Chem Biochem 1983;41:209-374.

20 Michalski JC, Strecker G, Fournet B, Cantz M, Spranger J. Structures of sialyloligosaccharides excreted in the urine of a patient with mucolipidosis I. FEBS Lett 1977;79:101-104.

21 Strecker G, Peers M-C, Michalski J-C, et al. Structures of nine sialyl-oligosaccharides accumulated in urine of eleven patients with three different types of sialidosis. Mucolipidosis II and two new types of mucolipidosis. Eur J Biochem 1977;75:391-403.

22 Dorland L, Haverkamp J, Vliegenthart JFG, et al. $360-\mathrm{MHz}^{1} \mathrm{H}$ nuclear-magnetic-resonance spectroscopy of sialyl-oligosaccharides from patients with sialidosis (mucolipidosis I and II). Eur J Biochem 1978;87:323-329.

23 Koseki M, Tsurumi K. Structure of a novel sialooligosaccharide from the urine of a patient with mucolipidosis. Tohoku J Exp Med 1978;124:361-366.

24 Koseki M, Tsurumi $\mathrm{K}$. The structure of a new sialic acid-containing decasaccharide from the urine of a patient with mucolipidosis. Tohoku J Exp Med 1979;128:39-49. 
25 Kuriyama M, Ariga T, Ando S, Suzuki M, Yamada T, Miyatake T. Four positional isomers of sialyloligosaccharides isolated from the urine of a patient with sialidosis. J Biol Chem 1981;256:12316-12321.

26 Kuriyama M, Ariga T, Ando S, et al. Two positional isomers of sialylheptasaccharides isolated from the urine of a patient with sialidosis. J Biochem 1985;98:1049-1054. 